\title{
Appearance and Disappearance of Quantum Correlations in Measurement-Based Feedback Control of a Mechanical Oscillator
}

\author{
V. Sudhir, ${ }^{1}$ D. J. Wilson, ${ }^{1}$ R. Schilling, ${ }^{1}$ H. Schütz, ${ }^{1}$ S. A. Fedorov, ${ }^{1}$ \\ A. H. Ghadimi, ${ }^{1}$ A. Nunnenkamp, ${ }^{2}$ and T. J. Kippenberg ${ }^{1, *}$ \\ ${ }^{1}$ Institute for Condensed Matter Physics, École Polytechnique Fédérale de Lausanne, \\ Lausanne 1015, Switzerland \\ ${ }^{2}$ Cavendish Laboratory, University of Cambridge, Cambridge CB3 OHE, United Kingdom
}

(Received 5 May 2016; revised manuscript received 1 November 2016; published 6 January 2017)

\begin{abstract}
Quantum correlations between imprecision and backaction are a hallmark of continuous linear measurements. Here, we study how measurement-based feedback can be used to improve the visibility of quantum correlations due to the interaction of a laser field with a nanomechanical oscillator. Backaction imparted by the meter laser, due to radiation-pressure quantum fluctuations, gives rise to correlations between its phase and amplitude quadratures. These quantum correlations are observed in the experiment both as squeezing of the meter field fluctuations below the vacuum level in a homodyne measurement and as sideband asymmetry in a heterodyne measurement, demonstrating the common origin of both phenomena. We show that quantum feedback, i.e., feedback that suppresses measurement backaction, can be used to increase the visibility of the sideband asymmetry ratio. In contrast, by operating the feedback loop in the regime of noise squashing, where the in-loop photocurrent variance is reduced below the vacuum level, the visibility of the sideband asymmetry is reduced. This is due to backaction arising from vacuum noise in the homodyne detector. These experiments demonstrate the possibility, as well as the fundamental limits, of measurement-based feedback as a tool to manipulate quantum correlations.
\end{abstract}

DOI: 10.1103/PhysRevX.7.011001

Measurements proceed by establishing correlations between a system and a meter. In a quantum description of this process [1], the effect of measurement persists in the system in the form of measurement backaction. For a class of measurements - continuous linear measurements $[2,3]$ — where the meter couples linearly and weakly to the system, correlations between the system and meter additionally manifest as backaction-induced quantum correlations between the degrees of freedom of the meter. A paradigmatic example is the interferometric position readout of a mechanical oscillator [4]. The meter in this case is an optical field, which possesses two degrees of freedom (quadratures): amplitude and phase. The position of the oscillator is imprinted onto the phase quadrature. Backaction arises from vacuum fluctuations of the amplitude quadrature, which are imprinted onto the phase via the backaction-driven motion of the oscillator, leading to amplitude-phase correlations in the meter field. In a homodyne detector, these quantum correlations manifest as ponderomotive squeezing of an appropriately chosen field quadrature [5-8]. In a heterodyne detector, they

\footnotetext{
* Corresponding author. tobias.kippenberg@epfl.ch

Published by the American Physical Society under the terms of the Creative Commons Attribution 4.0 International license. Further distribution of this work must maintain attribution to the author(s) and the published article's title, journal citation, and DOI.
}

Subject Areas: Quantum Physics

manifest as motional sideband asymmetry [9-12]. Differences between these effects arise from the details of how meter fluctuations are converted to a classical signal by the detection process [10,13-15] [16]

Here, we investigate the effect of measurement-based feedback on quantum correlations due to the interaction of an optical field with a nanomechanical oscillator. Recent advances [18] have enabled operation of an optomechanical system such that the mechanical oscillator can be measured at a rate approaching its thermal decoherence rate, a regime where measurement backaction becomes comparable to the thermal motion. Harnessing this capability, we show that feedback of a homodyne measurement can be used to improve the visibility of motional sideband asymmetry by suppressing both thermal motion and measurement backaction. Indeed, the feedback loop cools the oscillator to a final phonon occupancy $\left(n_{\text {eff }}\right)$ that is more than 2 orders of magnitude lower than that due to the quantum backaction $\left(n_{\mathrm{qba}}\right)$ of the meter beam. In contrast to previous work [19-30], our system therefore operates in the quantum feedback regime, where quantum backaction is effectively suppressed by feedback, and feedback can manipulate quantum correlations without destroying them. This is possible because the measurement used for feedback contains a faithful record of its own backaction [31]. Furthermore, we study how these quantum correlations are obscured in the regime where quantum noise in the in-loop detector causes the dominant force noise on the oscillator (i.e., feedback backaction): a regime corresponding to "squashing" of the in-loop photocurrent [32]. Conceptually, this feedback backaction-dominated regime is 
analogous to the quantum-backaction limit of sideband cooling [33]. Finally, we probe quantum correlations via a homodyne detector tuned close to the amplitude quadrature and observe squeezing, i.e., a reduction of the homodyne photocurrent noise below the vacuum level. By observing both squeezing and sideband asymmetry in the same field, the common origin of motional sideband asymmetry $[10,14]$ and ponderomotive squeezing $[8,11]$ in general linear detection of the meter field is experimentally illustrated.

A pedagogical description of continuous linear measurement is germane to understanding our approach. We denote as $x(t)$ the position of a quantum harmonic oscillator and $y(t) \propto x(t)$ the output of a linear continuous position detector. Since it is a continuous observable, $y(t)$ must commute with itself at different times $\left(\left[y(t), y\left(t^{\prime}\right)\right]=0\right)$. The physical motion $x(t)$ does not obey this constraint, which requires that the detector output contains an additional noise term $x_{\mathrm{n}}(t)$ that enforces the commutator. The noise $x_{\mathrm{n}}$ contains two components: an apparent (imprecision) noise, $x_{\text {imp }}$, which arises from quantum fluctuations of the meter degree of freedom coupled to the detector, and a physical (backaction) noise, $x_{\mathrm{ba}}$, which arises from quantum fluctuations of the meter degree of freedom coupled to the system. The total detector signal, $y=x+x_{\mathrm{ba}}+x_{\mathrm{imp}} \equiv x_{\text {tot }}+x_{\mathrm{imp}}$, is characterized by a (symmetrized, double-sided [34]) noise spectrum [2,3],

$$
\bar{S}_{y y}(\Omega)=\bar{S}_{x x}^{\mathrm{imp}}(\Omega)+\bar{S}_{x x}^{\mathrm{tot}}(\Omega)+2 \operatorname{Re} \bar{S}_{x_{\mathrm{ba}} x_{\mathrm{imp}}}(\Omega),
$$

which contains terms due to quantum fluctuations of the meter $\left(x_{\text {imp }}\right)$, total physical motion $\left(x_{\text {tot }}\right)$, and quantum (imprecision-backaction) correlations, respectively.

In our experiment, we monitor the position fluctuations of a cryogenically precooled $(T \approx 6 \mathrm{~K})$ nanomechanical string coupled dispersively to an optical microcavity [35]. The fundamental out-of-plane mode of the string forms the oscillator (frequency $\Omega_{\mathrm{m}}=2 \pi \cdot 4.3 \mathrm{MHz}$, damping rate $\Gamma_{m}=2 \pi \cdot 7 \mathrm{~Hz}$ ). The meter is a laser field passing resonantly through the cavity (wavelength $\lambda \approx 774 \mathrm{~nm}$ ), whose quadratures are monitored simultaneously by a homodyne and a heterodyne detector [Fig. 1(a)]. Both detectors are operated with an imprecision far below that at the standard quantum limit, implying that quantum backaction due to the measurement (quantified as a phonon occupancy $n_{\mathrm{qba}}$ ) contributes significantly to the total motion of the nanomechanical oscillator $\left(n_{\text {tot }}\right)$; in our case, $n_{\mathrm{qba}} \approx 0.15 n_{\text {tot }}$.

We first assess the resulting optomechanical quantum correlations by measuring the output field with a homodyne (a)

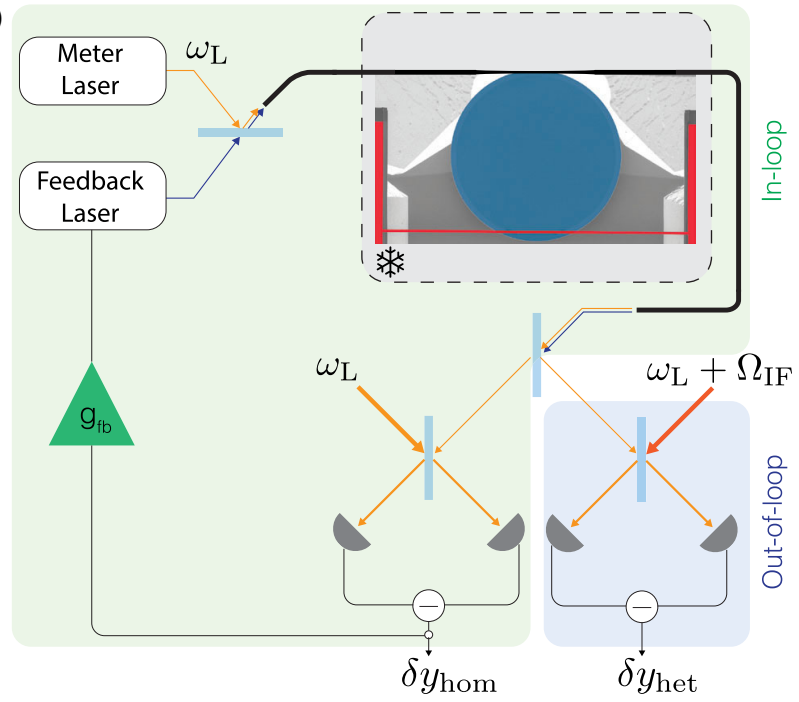

(b)

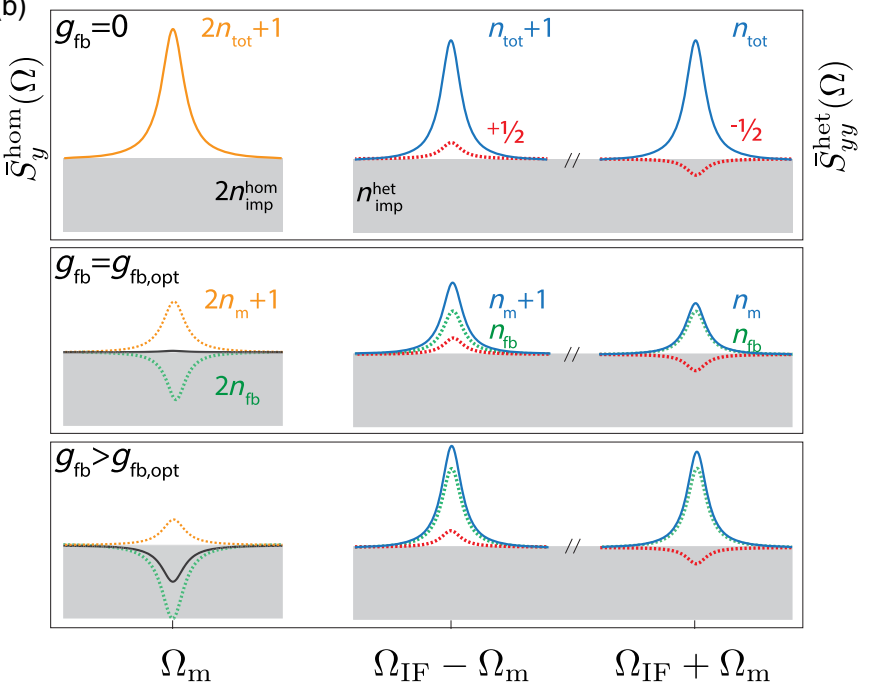

FIG. 1. Using homodyne feedback to increase the visibility of quantum-correlation-induced motional sideband asymmetry. (a) Linear position measurement and feedback control of a nanomechanical string $\left(\mathrm{Si}_{3} \mathrm{~N}_{4}\right.$, red) is provided by evanescent coupling to an optical microdisk cavity $\left(\mathrm{SiO}_{2}\right.$, blue). Whispering gallery modes of the microdisk are driven by a pair of tunable diode lasers using a tapered optical fiber (black). The "meter" field (orange arrow) is directed to a pair of balanced interferometers (homodyne, green; heterodyne, blue). A delayed and amplified copy of the homodyne signal is imprinted onto the amplitude of the "feedback" field (blue arrow), effecting cold damping of the fundamental beam mode. The taper, nanobeam, and microdisk are integrated into a He cryostat (grey). (b) Schematic of the closed-loop homodyne (left) and heterodyne (right) noise spectrum for various feedback gains. With no feedback $\left(g_{\mathrm{fb}}=0\right.$, top panel), the homodyne signal (orange line) is proportional to the total thermal occupation, while the heterodyne signal (blue line) is asymmetric because of $\pm \frac{1}{2}$ phonon equivalent contributions (red dashed line) from quantum correlations. At optimal feedback (middle panel), the homodyne signal (black line) coincides with the measurement imprecision (grey line) due to classical correlations (green dashed line) arising from feedback backaction exactly canceling the total motion (orange dashed line); in this case, visibility of heterodyne sideband asymmetry is maximum. A further increase in the feedback gain (bottom panel) leads to squashing of the homodyne signal and a decrease in the visibility of sideband asymmetry in the heterodyne detector due to feedback backaction that is large compared to the thermal occupation. 
detector. Measuring the quadrature of the meter field at phase $\theta$, imprecision-backaction correlations in the homodyne signal take the form [see Eq. (B8)]

$$
\bar{S}_{x_{\mathrm{ba}} x_{\mathrm{imp}}}^{\text {hom }}(\Omega) \propto C \eta_{\mathrm{hom}} \sin (2 \theta) \operatorname{Re} \chi_{m}(\Omega),
$$

where $C=4 g_{0}^{2} n_{c} / \kappa \Gamma_{m}$ is the multiphoton cooperativity of the optomechanical system, $\eta_{\text {hom }}$ is the detection efficiency, and $\chi_{m}(\Omega)=\left(-\Omega^{2}+\Omega_{m}^{2}-i \Omega \Gamma_{m}\right)^{-1} / m$ is the susceptibility of the mechanical oscillator to an applied force. Here, $g_{0}$ is the vacuum optomechanical coupling rate, $n_{c}$ is the mean intracavity photon number, and $\kappa\left(\Gamma_{\mathrm{m}}\right)$ is the cavity (oscillator) decay rate. When measuring the phase quadrature $(\theta=\pi / 2)$, where sensitivity to mechanical motion is largest [shown in Fig. 1(b), top left], these correlations do not appear in the homodyne photocurrent. However, near the amplitude quadrature $(\theta \rightarrow 0)$, the magnitude of the correlation term can be comparable to the thermal motion, leading to observable squeezing of the homodyne photocurrent [36]. Figure 2 shows homodyne detection of such optical squeezing near the amplitude quadrature. The observed squeezing, while small in magnitude $(\approx 1 \%)$, can still be clearly distinguished in the measurement.

Measurements of squeezing near the amplitude quadrature, reported in Fig. 2, are sensitive to laser amplitude noise [37], which in our experiments constitute less than $0.01 \%$ of the observed photocurrent shot noise (see Appendix A 2). In principle, the effect of laser phase noise is, on the one hand, negligible for amplitude quadrature squeezing, while on the other, it is suppressed heavily because of the bad cavity regime (i.e., $\Omega_{\mathrm{m}} \ll \kappa$ ) in which we operate. In practice, we verify that the squeezing spectrum is unchanged when using a low-noise Ti:Sa laser in lieu of a diode laser.

Detecting ponderomotive squeezing provides bona fide proof of the presence of quantum correlations in the meter field. Next, we probe the alternate manifestation of these correlations as sideband asymmetry-in a heterodyne detector. A heterodyne detector, as used in the experiment, monitors both quadratures of the meter simultaneously (see Appendix 1 a), giving access to $\bar{S}_{y y}^{\text {het }}(\Omega>0)$, where $\bar{S}_{y y}^{\text {het }}\left(\Omega_{\mathrm{IF}} \pm \Omega_{\mathrm{m}}\right)$ corresponds to upper $(+)$ and lower $(-)$ motional sidebands (displaced by the heterodyne intermediate frequency $\Omega_{\mathrm{IF}}$ ). Quantum correlations between the phase and amplitude of the meter manifest as an asymmetry of the heterodyne motional sidebands. This can be understood from the three terms in Eq. (1), illustrated as components of the heterodyne signal in Fig. 1(b) (top right panel). Detector imprecision (gray)—arising from the vacuum fluctuations in the phase and amplitude quadrature of the probe-contributes a phonon-equivalent noise of $n_{\mathrm{imp}}^{\text {het }} \equiv \bar{S}_{y y}^{\text {het,imp }}\left(\Omega_{\mathrm{IF}} \pm \Omega_{\mathrm{m}}\right) / \bar{S}_{x x}^{\mathrm{zp}}\left(\Omega_{\mathrm{m}}\right)$. Physical motion-arising from a combination of thermal force and meter backaction—contributes $n_{\mathrm{m}}+\frac{1}{2}$ phonons to each

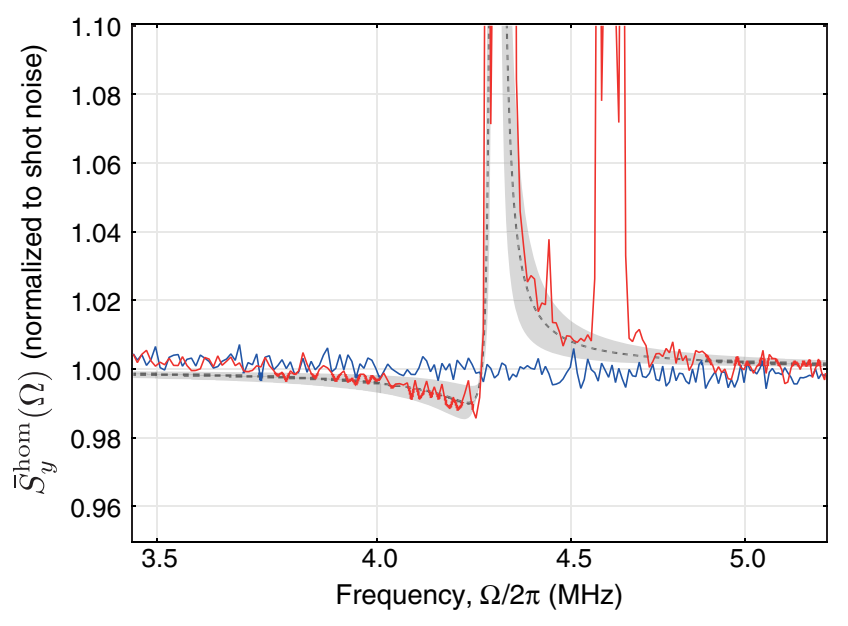

FIG. 2. Squeezing in homodyne detection. Quantum correlations in the meter field exiting the cavity manifest as squeezing when measured using a homodyne detector set near the amplitude quadrature. The blue trace shows the shot noise in the homodyne detector when the meter field is blocked. Red shows the measurement when the meter field has interacted with the mechanical oscillator; squeezing at the level of $1 \%$ is visible. Here, the homodyne interferometer, set close to the amplitude quadrature $(\theta \approx 0.15 \mathrm{rad})$, is fed with a quantum-noise-limited $\mathrm{Ti}: \mathrm{Sa}$ laser such that the optomechanical system is interrogated with a mean intracavity photon number, $n_{c} \approx 2 \times 10^{4}$. By directing all of the cavity transmission to the homodyne detector, we realize an overall detection efficiency of $\eta_{\text {hom }} \approx 0.2$. The observed squeezing agrees well with the theoretical prediction (black dashed line); the grey band shows models with $10 \%$ variation in $\theta, n_{c}, \eta_{\text {hom }}$. The wideband shot noise detected far from mechanical resonance agrees very well with the expected local oscillator shot noise. The peak at $\Omega \approx 2 \pi \times 4.6 \mathrm{MHz}$ is due to the in-plane mode of the nanobeam, whose contribution to the imprecision at $\Omega=\Omega_{\mathrm{m}} \approx 2 \pi \times 4.3 \mathrm{MHz}$ is negligible.

sideband. Imprecision-backaction correlations-arising from amplitude-phase correlations in the meter-contribute $\pm \frac{1}{2}$ phonons to the lower or upper sideband (red dashed line) [see Eq. (A23)]. Here $\bar{S}_{x x}^{z p}\left(\Omega_{\mathrm{m}}\right)=4 x_{\mathrm{zp}}^{2} / \Gamma_{\mathrm{m}}$ is the zeropoint position spectral density on resonance. The resulting asymmetry of the sidebands (blue traces),

$$
R \equiv \frac{\bar{S}_{y y}^{\text {het }}\left(\Omega_{\text {het }}^{+}\right)-\bar{S}_{y y}^{\text {het,imp }}\left(\Omega_{\text {het }}^{+}\right)}{\bar{S}_{y y}^{\text {het }}\left(\Omega_{\text {het }}^{-}\right)-\bar{S}_{y y}^{\text {het,imp }}\left(\Omega_{\text {het }}^{-}\right)} \approx \frac{n_{\mathrm{m}}}{n_{\mathrm{m}}+1},
$$

is commensurate with one phonon and arises purely from quantum correlations in the meter (here, $\Omega_{\text {het }}^{ \pm} \equiv \Omega_{\mathrm{IF}} \pm \Omega_{\mathrm{m}}$ ). This asymmetry corresponds directly to the visibility of imprecision-backaction correlations with respect to the total noise power, i.e.,

$$
\xi \equiv \frac{2 \operatorname{Re} \bar{S}_{x_{\mathrm{ba}} x_{\mathrm{imp}}}\left(\Omega_{\mathrm{het}}^{+}\right)}{\bar{S}_{x x}^{\mathrm{imp}}\left(\Omega_{\mathrm{het}}^{+}\right)+S_{x x}^{\mathrm{tot}}\left(\Omega_{\mathrm{het}}^{+}\right)} \approx \frac{1-R}{1+R}=\frac{1}{2 n_{\mathrm{m}}+1} .
$$


Our objective is to increase the sideband asymmetry $1-R$ in the heterodyne spectrum, and thereby $\xi$, by actively cold damping $[18,38]$ the mechanical oscillator using the homodyne measurement as an error signal. Concretely, the homodyne signal in the phase quadrature $(\theta=\pi / 2)$ is imprinted onto the amplitude quadrature of an independent feedback laser resonant with an auxiliary cavity mode $(\lambda \approx 840 \mathrm{~nm})$. The loop delay is tuned ( $\tau_{\mathrm{fb}} \approx 176 \mathrm{~ns}$, corresponding to a phase of $\phi_{\mathrm{fb}} \approx 3 \pi / 2$ at the mechanical frequency) in order to produce a purely viscous radiation pressure feedback force, effectively coupling the oscillator at a rate $\Gamma_{\mathrm{fb}} \approx g_{\mathrm{fb}} \Gamma_{m}$ to a cold bath with an occupation equal to the phonon-equivalent homodyne imprecision $n_{\mathrm{imp}}^{\text {hom }}=\bar{S}_{x}^{\text {imp,hom }}\left(\Omega_{\mathrm{m}}\right) / 2 \bar{S}_{x}^{\mathrm{zp}}\left(\Omega_{m}\right)$ (here, $g_{\mathrm{fb}}$ is the dimensionless gain of the feedback loop). The occupation of the oscillator is thereby reduced to $[18,38,39]$

$$
n_{\mathrm{m}}+\frac{1}{2} \approx \frac{n_{\mathrm{tot}}}{g_{\mathrm{fb}}}+g_{\mathrm{fb}} n_{\mathrm{imp}}^{\mathrm{hom}} \geq 2 \sqrt{n_{\mathrm{tot}} n_{\mathrm{imp}}^{\text {hom }}},
$$

with the minimum achieved at an optimal gain of $g_{\mathrm{fb}}^{\mathrm{opt}}=\sqrt{n_{\mathrm{tot}} / n_{\mathrm{imp}}^{\mathrm{hom}}}$. (Here, $n_{\mathrm{tot}}=n_{\mathrm{th}}+n_{\mathrm{ba}}$ is the effective bath occupation of the mechanical oscillator, including measurement backaction.) Notably, cold damping allows access to $n_{\mathrm{m}} \rightarrow 0$ when a highly efficient measurement is used, corresponding to an imprecision-backaction product approaching the uncertainty limit $2 \sqrt{n_{\mathrm{tot}} h_{\mathrm{imp}}^{\text {hom }}} \rightarrow \frac{1}{2}$. Two regimes may be identified: (1) an efficient feedback regime $\left(g_{\mathrm{fb}}<g_{\mathrm{fb}}^{\text {opt }}\right)$, in which the motion of the oscillatorresulting from thermal noise and measurement backaction-is efficiently suppressed; (2) an inefficient feedback regime, in which thermal force and measurement backaction are overwhelmed by feedback backaction $n_{\mathrm{fb}}=g_{\mathrm{fb}}^{2} n_{\mathrm{imp}}^{\text {hom }}$ (i.e., feedback of homodyne imprecision noise), resulting in an increase of $n_{\mathrm{m}}$. We explore these regimes in two experiments.

An experimental demonstration of efficient feedback cooling, where feedback backaction is weak $\left(n_{\mathrm{fb}}<n_{\mathrm{tot}}\right)$, is shown in Fig. 3. Here, $n_{\text {tot }} \approx 7 \times 10^{4}$, corresponding to an effective bath temperature of $13 \mathrm{~K}$ (arising partly because of quantum measurement backaction, $n_{\mathrm{ba}} \approx 4 \times 10^{4}$ [18]). From the perspective of the heterodyne measurement, the objective is to "distill" a motional sideband asymmetry of one phonon out of $n_{\mathrm{tot}}$. This is made possible by a low shotnoise-limited homodyne imprecision of $n_{\mathrm{imp}}^{\text {hom }} \approx 1.2 \times 10^{-4}$ [see Fig. 3(d) for details]. To trace out the cooling curve in Fig. 3(a), the feedback gain is tuned electronically while keeping all other experimental parameters (such as mean optical power and laser-cavity detuning) fixed. The sideband ratio $R$ is extracted from fitting a Lorentzian to each heterodyne sideband and taking the ratio of the fitted areas. The phonon occupation $n_{\mathrm{m}}$ is inferred from $R$ as well as the area beneath the lower sideband. In-loop (homodyne) and out-of-loop (heterodyne) noise spectra are shown in Fig. 3(b). As a characteristic of the efficient feedback regime, the area under the left sideband decreases linearly with $g_{\mathrm{fb}}$, corresponding to $n_{m} \propto g_{\mathrm{fb}}^{-1}$ [red circles in Fig. 3(a)]. As the optimal gain is approached, the in-loop spectrum is reduced to the imprecision noise floor [black trace in Fig. 3(b)]. This transition coincides with the "appearance" of a sideband asymmetry of $1-R \approx 12 \% \quad(\xi \approx 6 \%)$, corresponding to $n_{\mathrm{m}} \approx 7.3$.

To confirm the faithfulness of these measurements, two major sources of error were investigated:

(1) Drift over the course of measurement can introduce small changes in the relative magnitude of $\bar{S}_{y y}^{\text {het }}\left(\Omega_{\text {het }}^{ \pm}\right)$. In our experiment, this effect is mitigated by recording both heterodyne sidebands simultaneously. Augmented by operating in the bad cavity regime $\left(\Omega_{m} / \kappa \ll 10^{-3}\right)$ and the exceptionally low imprecision of the heterodyne measurement, $n_{\text {imp }}^{\text {het }}=\left(4 \eta_{\text {het }} C_{0} n_{c}\right)^{-1} \approx 3 \times 10^{-3}$ [see Fig. 3(d)], statistical fluctuations of $R$ over the course of a typical measurement set can be as small as $0.5 \%$ [see Fig. 3(c)]. Error bars for $R$ in Fig. 3(a) are derived from the standard deviation of similar data sets, in addition to a small contribution from the fit covariance matrix. At the largest damping rates, the reduced heterodyne signal-to-noise ratio results in insufficient convergence of the periodogram estimate of the spectra (keeping acquisition time and analysis bandwidth fixed), leading to larger error bars, $\delta R= \pm 2 \%$.

(2) Excess laser noise affects $R$ by producing additional imprecision-backaction correlations [10,37]. Assuming a mean thermal photon occupation of $C_{q q(p p)}$ for the amplitude (phase) quadrature of the injected meter field, the correlator in Eq. (1) becomes [see Eq. (A26)]

$$
\frac{2 \operatorname{Re} \bar{S}_{x_{\mathrm{b}} x_{\mathrm{imp}}}^{\text {het }}\left(\Omega_{\mathrm{het}}^{ \pm}\right)}{\bar{S}_{x x}^{\mathrm{zp}}\left(\Omega_{\mathrm{m}}\right)}=\mp \eta_{\mathrm{het}}\left(\frac{1}{2}+C_{q q} \pm \frac{4 \bar{\Delta} \Omega_{m}}{\kappa^{2}} C_{p p}\right),
$$

where $\eta_{\text {het }}$ is the heterodyne detection efficiency, and $\bar{\Delta}$ is the mean laser-cavity detuning. In our experiment, independent measurements reveal that $C_{q q}<0.01$ and $C_{p p}<30$ (owing partly to excess cavity frequency noise) for typical meter powers of $P_{\text {in }}<5 \mu \mathrm{W}$ (see Appendix A 2). Operating on resonance $(\bar{\Delta} \approx 0)$ and in the bad cavity regime substantially reduces sensitivity to $C_{p p}$. Using a typical value of $\bar{\Delta}=0.01 \times \kappa$, we estimate that $\left[\left(4 \bar{\Delta} \Omega_{m}\right) / \kappa^{2}\right] C_{p p}<0.005$, contributing negligibly to Eq. (6). Operating in the bad cavity regime also alleviates systematics arising from an asymmetric 

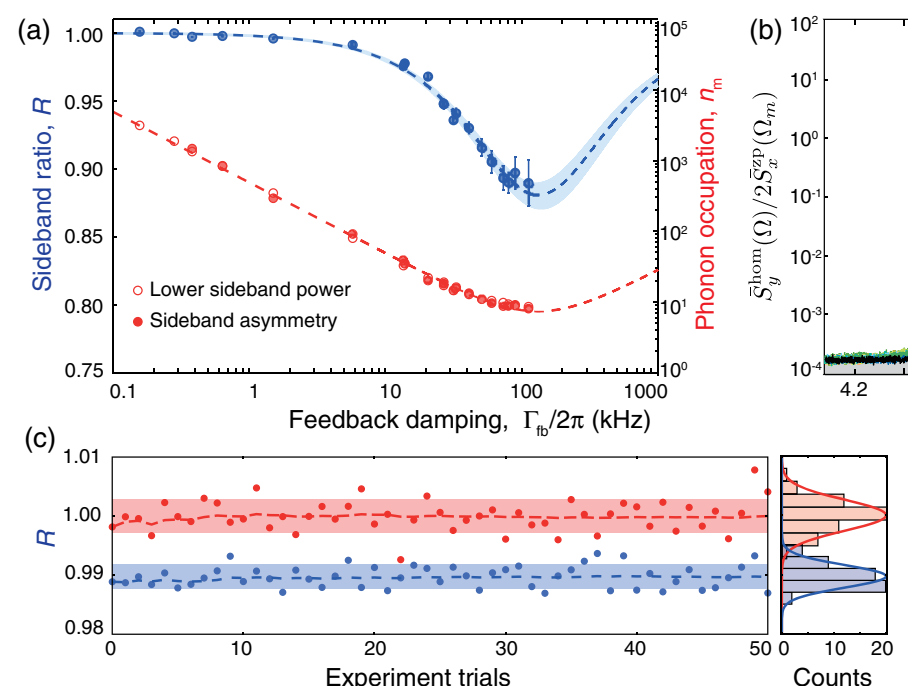

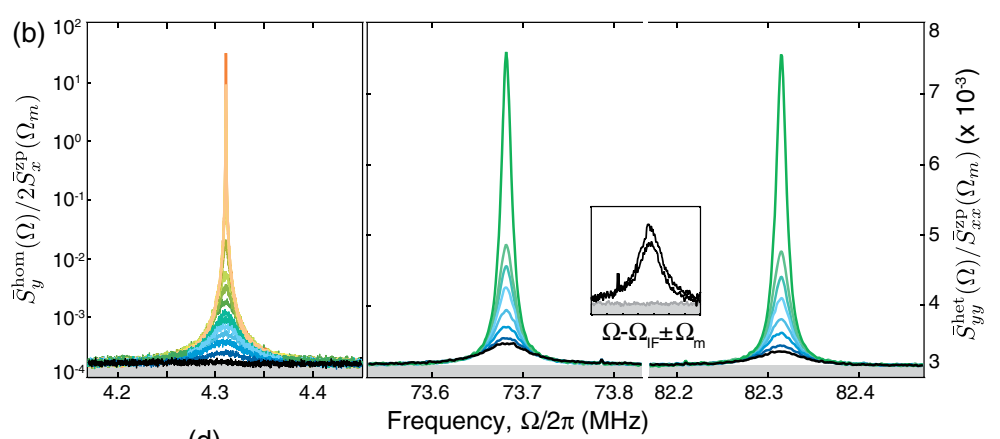

(d)

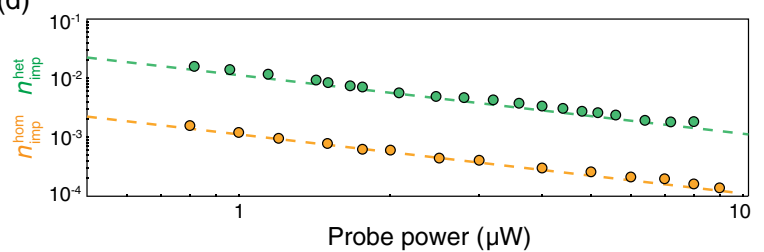

FIG. 3. Motional sideband asymmetry in the heterodyne measurement of a cold-damped mechanical oscillator. (a) Heterodyne sideband asymmetry $\left(R\right.$, blue) and inferred mechanical mode occupation $\left(n_{\mathrm{m}}\right.$, red) vs closed-loop mechanical damping rate $\left(\Gamma_{\mathrm{fb}}\right)$ for various feedback gains. A maximum asymmetry of $1-R \approx 12 \%\left(n_{\mathrm{m}} \approx 7.3\right)$ appears as the feedback gain approaches its optimal value. Dashed lines correspond to models $R=n_{\mathrm{m}} /\left(n_{\mathrm{m}}+1\right)$ [Eq. (A25), blue line] and $n_{\mathrm{m}}+\frac{1}{2} \approx\left(\Gamma_{\mathrm{m}} / \Gamma_{\mathrm{fb}}\right) n_{\mathrm{tot}}+\left(\Gamma_{\mathrm{fb}} / \Gamma_{\mathrm{m}}\right) n_{\mathrm{imp}}^{\text {hom }}$ [Eq. (5), red line]. The solid blue band is a confidence interval based on uncertainties in estimates of $n_{\text {tot }}, n_{\mathrm{imp}}^{\text {hom }}$, and $\Gamma_{\mathrm{m}}$. We infer the feedback damping rate $\Gamma_{\mathrm{fb}}$ from the observed linewidth of the out-of-loop spectra, given by $\Gamma_{\mathrm{m}}+\Gamma_{\mathrm{fb}}$, and the known value of the intrinsic linewidth $\Gamma_{\mathrm{m}} \approx 2 \pi \times 7 \mathrm{~Hz}$ measured independently. Open red circles are independent estimates of $n_{\mathrm{m}}$ based on the area beneath the left heterodyne sideband. (b) Homodyne (left panel) and heterodyne (right panel) spectra used to obtain (a). Black traces correspond to lowest occupation; asymmetry is highlighted in the inset. Only a subset of heterodyne spectra are shown, for low $n_{\mathrm{m}}$, with colors matching the corresponding homodyne spectra. An important feature of these spectra is their low imprecision noise floor, $n_{\mathrm{imp}}^{\text {hom }}=$ $\left(16 \eta_{\text {hom }} C_{0} n_{\mathrm{c}}\right)^{-1}=1.2 \times 10^{-4}$ and $n_{\text {imp }}^{\text {het }}=\left(4 \eta_{\text {het }} C_{0} n_{c}\right)^{-1}=2.9 \times 10^{-3}$. This is made possible by the high photon collection efficiency $\eta \approx 0.2$, single photon cooperativity, $C_{0}=4 g_{0}^{2} / \kappa \Gamma_{\mathrm{m}} \approx 0.3$, and power handling capacity of the microcavity-based sensor (allowing for intracavity photon numbers of $n_{\mathrm{c}} \approx 10^{4}$ ). (c) Statistical fluctuations of $R$ for low feedback gain, indicates the ability to discriminate a $0.5 \%$ asymmetry, corresponding to $n_{\mathrm{m}} \approx 100$. (d) Phonon-equivalent imprecision of the heterodyne and homodyne detectors as a function of the power of the meter field, showing the linear scaling expected for quantum-noise-limited operation.

cavity transduction when $\bar{\Delta} \neq 0$ : For a finite detuning offset, the apparent asymmetry is given by $4 \bar{\Delta} \Omega_{m} / \kappa^{2}$, which is typically less than $0.1 \%$ in our experiments.

Having established that our measurements of motional sideband asymmetry are not contaminated by classical artifacts, the results shown in Fig. 3 may be interpreted as "distillation" of quantum correlations using feedback. Efficient measurement-based feedback can therefore coherently modify the response of the optomechanical system while suppressing quantum backaction but not destroying the subtle correlations they establish in the meter.

We now explore the complementary regime of inefficient feedback, where feedback backaction is stronger than the thermal force and measurement backaction $\left(n_{\mathrm{fb}}>n_{\mathrm{tot}}\right)$. We access this regime by changing the homodyne/heterodyne splitting ratio, thereby increasing the homodyne imprecision to $n_{\mathrm{imp}}^{\text {hom }} \approx 10^{-3}$. As shown in Fig. 4, increasing the gain beyond its optimum value (corresponding to $n_{\mathrm{m}} \approx 13.4$ and $1-R \approx 7 \%$ ) results in a reduction of the homodyne signal below the shot-noise level [Fig. 4(b), left panel]. Simultaneously, the areas of the heterodyne sidebands increase, while their asymmetry $(1-R)$ decreases. The discrepancy between "squashing" $[32,40]$ of the in-loop signal and the "disappearance" of sideband asymmetry relates to a basic difference between feedback backaction and meter backaction; namely, feedback backaction is correlated with the in-loop imprecision and not with the out-of-loop imprecision [32].

Squashing of the in-loop signal is caused by correlations between the feedback backaction-driven motion $x_{\mathrm{fb}}$ and the in-loop measurement imprecision [see Eq. (C2)],

$$
\frac{2 \operatorname{Re} \bar{S}_{x_{\mathrm{f}} x_{\mathrm{imp}}}^{\mathrm{hom}}\left(\Omega_{m}\right)}{2 \bar{S}_{x x}^{\mathrm{zp}}\left(\Omega_{m}\right)}=-n_{\mathrm{imp}}^{\mathrm{hom}} g_{\mathrm{fb}},
$$

represented by the negative-valued green trace in Fig. 1(b) (left panel). Interestingly, these classical correlations, in conjunction with the generalized Heisenberg uncertainty principle $[2,3]$, can be used to predict the transition from efficient to inefficient feedback; viz.

$$
\bar{S}_{F F} \cdot \bar{S}_{x x}^{\text {imp,hom }} \geq \frac{\hbar^{2}}{2}+\left(2 \operatorname{Re} \bar{S}_{F x_{\text {imp.hom }}}\right)^{2}
$$



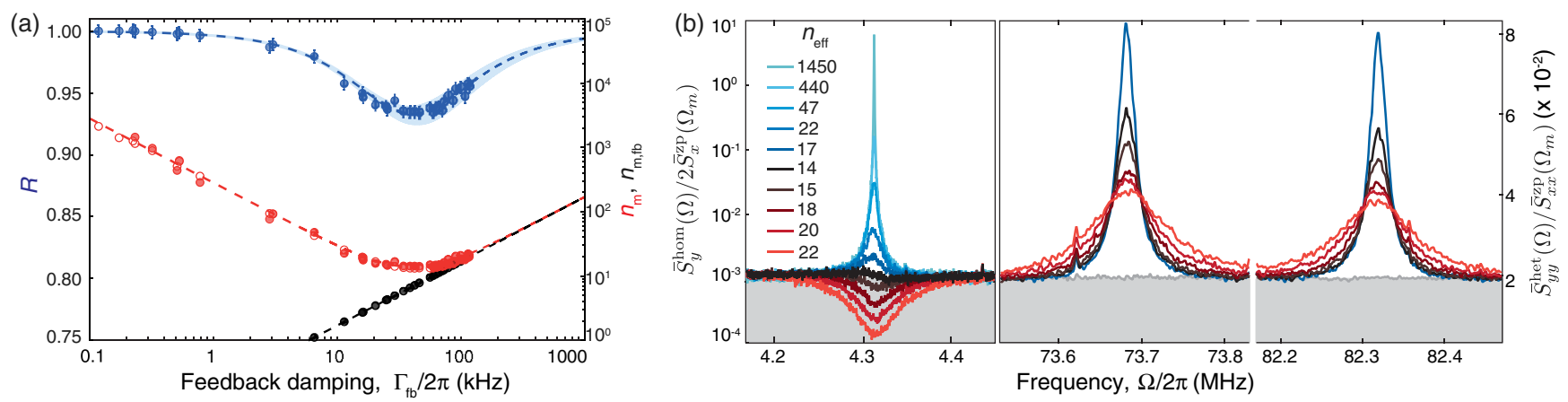

FIG. 4. Appearance and disappearance of sideband asymmetry. (a) Repeat of the experiment shown in Fig. 3(a) with lower homodyne detection efficiency. Feedback with the same range of gain results in lower optimal asymmetry $(R \approx 6 \%)$ and accesses the "strong feedback" regime in which feedback backaction $\left(n_{\mathrm{fb}}\right)$ dominates physical motion, resulting in reduced $R$. Black points are an estimate of the mechanical occupation due to feedback backaction, $n_{\mathrm{m}, \mathrm{fb}}=\left(\Gamma_{m} / \Gamma_{\mathrm{fb}}\right) n_{\mathrm{fb}}=g_{\mathrm{fb}} n_{\mathrm{imp}}^{\text {hom }}$, based on the noise floor of the homodyne spectra. (b) Left panel: In-loop homodyne spectra. In the strong feedback regime, noise is "squashed" (reduced below the open-loop imprecision), corresponding to in-loop squeezing. Right panel: Out-of-loop heterodyne spectra. Inefficient feedback manifests as an increase in the off-resonant noise power and reduced asymmetry.

is saturated for $g_{\mathrm{fb}}^{\mathrm{opt}}=\sqrt{n_{\mathrm{tot}} / n_{\mathrm{imp}}^{\text {hom }}}$ [using $F_{\mathrm{fb}} \propto g_{\mathrm{fb}} x_{\mathrm{imp}}^{\text {hom }}$ and Eq. (7)]. The limits of feedback cooling and the prospects for feedback-based enhancement of quantum correlations are related to the detection of meter fluctuations and the choice of feedback strategy — optimization of either seems pertinent.

Looking forward, efficient measurement-based feedback may be directly compared against autonomous control for the manipulation of quantum systems. For example, unconditional squeezing of a mechanical oscillator may be realized from a record of the backaction evasion measurement of its motion [41]. Ultimately, with a sufficiently efficient linear measurement, a harmonic oscillator may be rendered anharmonic by nonlinear feedback.

\section{ACKNOWLEDGMENTS}

All samples are fabricated at the CMi (Center for MicroNanoTechnology) at EPFL. Research is funded by an ERC Advanced Grant (QuREM), a Marie Curie Initial Training Network Cavity Quantum Optomechanics, the Swiss National Science Foundation, and through support from the NCCR of Quantum Engineering (QSIT). D. J. W. acknowledges support from the European Commission through a Marie Curie Fellowship (IIF Project No. 331985).

\section{APPENDIX A: EXCESS LASER NOISE}

The effect of laser noise on sideband asymmetry measurements is well studied for cavity optomechanical systems in the resolved sideband regime [37,42]. In this case, sidebands have been observed separately by scattering them into the cavity with a probe laser red/blue detuned. Here, we discuss the effect of laser noise on sideband asymmetry measurements in the "bad cavity" regime $\left(\Omega_{m} \ll \kappa\right)$, wherein a resonant probe is used to detect the sidebands simultaneously in a heterodyne measurement. A theoretical model is developed in Sec. A 1. In Sec. A 2, we present measurements confirming the negligible contribution of laser noise to the reported results.

\section{Contribution of excess noise for resonant probing and simultaneous detection of sidebands}

In our experiment, we probe the optomechanical system using a resonant laser at frequency $\omega_{L}$. The photon flux amplitude operator of the laser, $a_{\mathrm{in}}(t)$, is assumed to have the form

$$
a_{\text {in }}(t)=e^{-i \omega_{L} t}\left[\bar{a}_{\text {in }}+\delta a_{\text {in }}(t)\right],
$$

where $\bar{a}_{\text {in }}=\sqrt{P_{\text {in }} / \hbar \omega_{L}}$ is the mean photon flux and the fluctuations $\delta a_{\text {in }}(t)$ satisfy

$$
\left[\delta a_{\mathrm{in}}(t), \delta a_{\mathrm{in}}^{\dagger}\left(t^{\prime}\right)\right]=\alpha \delta\left(t-t^{\prime}\right) .
$$

Note that we explicitly "tag" the commutator so as to follow its contribution to the measured quantities [10]; in reality, $\alpha=1$.

The canonically conjugate quadratures corresponding to the fluctuations are defined as

$$
\begin{aligned}
\delta q_{\mathrm{in}}(t) & :=\frac{\delta a_{\mathrm{in}}(t)+\delta a_{\mathrm{in}}^{\dagger}(t)}{\sqrt{2}}, \\
\delta p_{\mathrm{in}}(t) & :=\frac{\delta a_{\mathrm{in}}(t)-\delta a_{\mathrm{in}}^{\dagger}(t)}{i \sqrt{2}},
\end{aligned}
$$

such that

$$
\left[\delta q_{\text {in }}(t), \delta p_{\text {in }}\left(t^{\prime}\right)\right]=i \alpha \delta\left(t-t^{\prime}\right) .
$$

Excess ("classical") noise in the laser is modeled as Gaussian fluctuations, for which 


$$
\begin{aligned}
& \left(\begin{array}{cc}
\left\langle\delta q_{\text {in }}(t) \delta q_{\text {in }}\left(t^{\prime}\right)\right\rangle & \left\langle\delta q_{\text {in }}(t) \delta p_{\text {in }}\left(t^{\prime}\right)\right\rangle \\
\left\langle\delta p_{\text {in }}(t) \delta q_{\text {in }}\left(t^{\prime}\right)\right\rangle & \left\langle\delta p(t) \delta p\left(t^{\prime}\right)\right\rangle
\end{array}\right) \\
& =\frac{1}{2}\left(\begin{array}{cc}
\alpha+2 C_{q q} & i \alpha+2 C_{q p} \\
-i \alpha+2 C_{q p} & \alpha+2 C_{p p}
\end{array}\right) \delta\left(t-t^{\prime}\right) .
\end{aligned}
$$

The terms $C_{i j}(i=q, p)$ represent the noise in excess of the fundamental vacuum fluctuations in the field quadratures, distributed uniformly (i.e., "white") in frequency. We henceforth omit the cross-correlation $C_{q p}$ and attempt to bound its effect via an appropriate inequality [43] (see Sec. A 3). Thus,

$$
\begin{gathered}
\left(\begin{array}{cc}
\left\langle\delta a_{\mathrm{in}}(t) \delta a_{\mathrm{in}}\left(t^{\prime}\right)\right\rangle & \left\langle\delta a_{\mathrm{in}}(t) \delta a_{\mathrm{in}}^{\dagger}\left(t^{\prime}\right)\right\rangle \\
\left\langle\delta a_{\mathrm{in}}^{\dagger}(t) \delta a_{\mathrm{in}}\left(t^{\prime}\right)\right\rangle & \left\langle\delta a_{\mathrm{in}}^{\dagger}(t) \delta a_{\mathrm{in}}^{\dagger}\left(t^{\prime}\right)\right\rangle
\end{array}\right) \\
=\frac{1}{2}\left(\begin{array}{cc}
C_{q q}-C_{p p} & 2 \alpha+C_{q q}+C_{p p} \\
C_{q q}+C_{p p} & C_{q q}-C_{p p}
\end{array}\right) .
\end{gathered}
$$

We now consider an optomechanical system where the optical cavity is driven by a noisy input field satisfying Eq. (A6). The mechanical oscillator couples to the cavity field via radiation pressure and is additionally driven by a thermal Langevin force. Fluctuations of the intracavity field amplitude $(\delta a)$ and the mechanical oscillator amplitude $(\delta b)$ around their stable steady states satisfy [47]

$$
\begin{gathered}
\dot{\delta a}=+i \Delta \delta a-\frac{\kappa}{2} \delta a+i g\left(\delta b+\delta b^{\dagger}\right)+\sqrt{\kappa} \delta a_{\mathrm{in}}, \\
\dot{\delta b}=-i \Omega_{m} \delta b-\frac{\Gamma_{m}}{2} \delta b+i\left(g^{\star} \delta a+g \delta a^{\dagger}\right)+\sqrt{\Gamma_{m}} \delta b_{\mathrm{in}} .
\end{gathered}
$$

Here, $\Delta=\omega_{L}-\omega_{c}$ is the laser detuning, $g=g_{0} \bar{a}$ is the dressed ("multiphoton") optomechanical coupling rate, and $\bar{a}=\sqrt{\kappa} \bar{a}_{\text {in }} /(\kappa / 2)-i \Delta$ is the mean intracavity field amplitude. We have also assumed here that the cavity decay rate is dominated by its external coupling, i.e., $\kappa=\kappa_{0}+\kappa_{\mathrm{ex}} \approx \kappa_{\mathrm{ex}}$. The mechanical Langevin noise correlators are

$$
\begin{gathered}
\left\langle\delta b_{\text {in }}(t) \delta b_{\text {in }}^{\dagger}\left(t^{\prime}\right)\right\rangle=\left(n_{\text {th }}+\beta\right) \delta\left(t-t^{\prime}\right), \\
\left\langle\delta b_{\text {in }}^{\dagger}(t) \delta b_{\text {in }}\left(t^{\prime}\right)\right\rangle=n_{\text {th }} \delta\left(t-t^{\prime}\right),
\end{gathered}
$$

where $n_{\text {th }}$ is the ambient mean thermal phonon occupation of the oscillator. Note that we also "tag" the contribution due to the zero-point fluctuation of the thermal bath to determine its role in the observables; in reality, $\beta=1$.

Equations (A7) and (A8) can be solved in the Fourier domain,

$$
\begin{aligned}
\delta a[\Omega]= & \chi_{c}[\Omega]\left[\sqrt{\kappa} \delta a_{\mathrm{in}}[\Omega]+i g\left(\delta b[\Omega]+\delta b^{\dagger}[\Omega]\right)\right], \\
\delta a^{\dagger}[\Omega]= & \delta a[-\Omega]^{\dagger}=\chi_{c}^{\star}[-\Omega]\left[\sqrt{\kappa} \delta a_{\mathrm{in}}^{\dagger}[\Omega]-i g^{\star}\left(\delta b[\Omega]+\delta b^{\dagger}[\Omega]\right)\right], \\
\left(\begin{array}{c}
\delta b[\Omega] \\
\delta b^{\dagger}[\Omega]
\end{array}\right)= & \frac{\sqrt{\Gamma_{m}}}{\mathcal{N}[\Omega]}\left(\begin{array}{cc}
\chi_{m}^{\star-1}[-\Omega]-i \Sigma[\Omega] & -i \Sigma[\Omega] \\
+i \Sigma[\Omega] & \chi_{m}^{-1}[\Omega]+i \Sigma[\Omega]
\end{array}\right)\left(\begin{array}{c}
\delta b_{\text {in }}[\Omega] \\
\delta b_{\text {in }}^{\dagger}[\Omega]
\end{array}\right) \\
& +\frac{i \sqrt{\kappa}}{\mathcal{N}[\Omega]}\left(\begin{array}{cc}
g^{\star} \chi_{m}^{\star-1}[-\Omega] \chi_{c}[\Omega] & g \chi_{m}^{\star-1}[-\Omega] \chi_{c}^{\star}[-\Omega] \\
-g^{\star} \chi_{m}^{-1}[\Omega] \chi_{c}[\Omega] & -g \chi_{m}^{-1}[\Omega] \chi_{c}^{\star}[-\Omega]
\end{array}\right)\left(\begin{array}{c}
\delta a_{\text {in }}[\Omega] \\
\delta a_{\text {in }}^{\dagger}[\Omega]
\end{array}\right) .
\end{aligned}
$$

Here, $\chi_{m}$ and $\chi_{c}$ are the bare mechanical and cavity response functions, respectively, given by

$$
\begin{aligned}
\chi_{m}[\Omega] & :=\left[\Gamma_{m} / 2-i\left(\Omega-\Omega_{m}\right)\right]^{-1}, \\
\chi_{c}[\Omega] & :=[\kappa / 2-i(\Omega+\Delta)]^{-1} .
\end{aligned}
$$

Note that $\Sigma[\Omega]$ is the mechanical "self-energy,"

$$
\Sigma[\Omega]=-i|g|^{2}\left(\chi_{c}[\Omega]-\chi_{c}^{\star}[-\Omega]\right)=\Sigma^{\star}[-\Omega],
$$

which describes the modification to the mechanical response due to radiation pressure, and

$$
\mathcal{N}[\Omega]=\chi_{m}^{-1}[\Omega] \chi_{m}^{\star-1}[-\Omega]+2 \Omega_{m} \Sigma[\Omega]=\mathcal{N}^{\star}[-\Omega] .
$$

The input-output relation, $\delta a_{\text {out }}=\delta a_{\text {in }}-\sqrt{\kappa} \delta a$, gives the fluctuations of the output fields in terms of the fluctuations of the input fields:

$$
\delta a_{\mathrm{out}}=A[\Omega] \delta a_{\mathrm{in}}+B[\Omega] \delta a_{\mathrm{in}}^{\dagger}+C[\Omega] \delta b_{\mathrm{in}}+D[\Omega] \delta b_{\mathrm{in}}^{\dagger},
$$

where

$$
\begin{aligned}
A[\Omega] & =1-\kappa \chi_{c}[\Omega]-\frac{2 i|g|^{2} \kappa \Omega_{m} \chi_{c}[\Omega]^{2}}{\mathcal{N}[\Omega]} \\
& \approx-\left(1+4 i \frac{\Delta}{\kappa}\right)\left(1+C_{0} n_{c} \frac{2 i \Omega_{m} \Gamma_{m}}{\mathcal{N}[\Omega]}\right), \\
B[\Omega] & =-\frac{2 i g^{2} \kappa \Omega_{m} \chi_{c}[\Omega] \chi_{c}^{\star}[-\Omega]}{\mathcal{N}[\Omega]} \approx-C_{0} n_{c} \frac{2 i \Omega_{m} \Gamma_{m}}{\mathcal{N}[\Omega]}, \\
C[\Omega] & =-\frac{i g \sqrt{\kappa \Gamma_{m}}}{\mathcal{N}[\Omega]} \chi_{c}[\Omega] \chi_{m}^{\star-1}[-\Omega] \\
& \approx-i \sqrt{C_{0} n_{c}}\left(1+2 i \frac{\Delta}{\kappa}\right) \Gamma_{m} \chi_{m}[\Omega], \\
D[\Omega] & =-\frac{i g \sqrt{\kappa \Gamma_{m}}}{\mathcal{N}[\Omega]} \chi_{c}[\Omega] \chi_{m}^{-1}[\Omega] \\
& \approx-i \sqrt{C_{0} n_{c}}\left(1+2 i \frac{\Delta}{\kappa}\right) \Gamma_{m} \chi_{m}^{\star}[-\Omega] .
\end{aligned}
$$


Here, approximate expressions are given for the case of interest, namely, resonant probing $(|\Delta| \ll \kappa)$, small sideband resolution $\left(\Omega_{m} \ll \kappa\right)$, and weak coupling $(|g| \ll \kappa)$. We have also introduced the single-photon cooperativity, $C_{0}=4 g_{0}^{2} /\left(\kappa \Gamma_{m}\right)$, and the mean intracavity photon number, $n_{c}=|\bar{a}|^{2}$.

Balanced heterodyne detection of the cavity output is used to measure motional sideband asymmetry. The output field is superposed on a balanced beam splitter with a frequency-shifted local oscillator,

$$
a_{\mathrm{LO}}=e^{-i\left(\omega_{\mathrm{L}}+\Omega_{\mathrm{IF}}\right) t}\left(\bar{a}_{\mathrm{LO}}+\delta a_{\mathrm{LO}}\right) .
$$

The fields at the output of the beam splitter, $\frac{1}{\sqrt{2}}\left(a_{\mathrm{LO}} \pm a_{\text {out }}\right)$, are detected with identical square-law detectors, whose photocurrents are subtracted. Note the implicit assumption that the local oscillator and signal paths are balanced in length; together with a balance of power beyond the combining beam splitter, this ensures suppression of common-mode excess noise [13].

The difference photocurrent is described by the operator

$$
I \propto a_{\mathrm{LO}}^{\dagger} a_{\mathrm{out}}+\text { H.c. }
$$

When $\bar{a}_{\mathrm{LO}} \gg \bar{a}_{\text {out }}$, fluctuations in the photocurrent are described by

$$
\delta I(t) \propto e^{-i \Omega_{\mathrm{IF}} t} \bar{a}_{\mathrm{LO}}^{\star} \delta a_{\mathrm{out}}(t)+\text { H.c. }
$$

The power spectrum of the heterodyne photocurrent is proportional to

$$
\bar{S}_{I I}^{\text {het }}(\Omega)=\frac{1}{2} \int_{-\infty}^{\infty}\left\langle\overline{\left\{\delta I\left(t+t^{\prime}\right), \delta I\left(t^{\prime}\right)\right\}}\right\rangle e^{i \Omega t} d t,
$$

where we have introduced the (time-averaged) current correlator,

$$
\begin{aligned}
\overline{\left\{\delta I\left(t+t^{\prime}\right), \delta I\left(t^{\prime}\right)\right\}} \propto & e^{-i \Omega_{\mathrm{IF}} t}\left\{\delta a_{\mathrm{out}}^{\dagger}(t), \delta a_{\text {out }}(0)\right\} \\
& +e^{+i \Omega_{\mathrm{IF}} t}\left\{\delta a_{\text {out }}(t), \delta a_{\text {out }}^{\dagger}(0)\right\}
\end{aligned}
$$

Assuming $\Omega_{\mathrm{IF}} \gg \Omega_{m}>0$, we obtain, for the balanced heterodyne spectrum normalized to the local oscillator shot noise,

$$
\begin{aligned}
\bar{S}_{I I}^{\text {het }}\left(\Omega-\Omega_{\mathrm{IF}}\right) & \approx \alpha+4 C_{0} n_{c} \frac{\Gamma_{m}^{2}}{4}\left[\left|\chi_{m}[-\Omega]\right|^{2}\left(n_{\mathrm{tot}}+\frac{\beta}{2}-\left(\frac{\alpha}{2}+C_{q q}\right)+\frac{4 \Delta \Omega_{m}}{\kappa^{2}} C_{p p}\right)\right. \\
& \left.+\left|\chi_{m}[\Omega]\right|^{2}\left(n_{\mathrm{tot}}+\frac{\beta}{2}+\left(\frac{\alpha}{2}+C_{q q}\right)+\frac{4 \Delta \Omega_{m}}{\kappa^{2}} C_{p p}\right)\right] .
\end{aligned}
$$

This represents the heterodyne spectrum measured in the experiment and depicted in Figs. 3 and 4 . Here, the total bath occupation, arising from the ambient thermal bath and the measurement backaction due to the meter beam, is given by

$$
n_{\mathrm{tot}}=n_{\mathrm{th}}+\underbrace{C_{0} n_{c}\left(\alpha+C_{q q}+\left(\frac{4 \Delta \Omega_{m}}{\kappa^{2}}\right)^{2} C_{p p}\right)}_{n_{\mathrm{ba}}} .
$$

The sideband ratio extracted from such a spectrum is

$$
\begin{aligned}
R & :=\frac{\int_{0^{+}}^{+\infty}\left(\bar{S}_{I I}^{\text {het }}\left(\Omega-\Omega_{\mathrm{IF}}\right)-\bar{S}_{I I}^{\text {het }}\left(\Omega=\Omega_{\mathrm{IF}}^{+}\right)\right) \frac{d \Omega}{2 \pi}}{\int_{-\infty}^{0^{-}}\left(\bar{S}_{I I}^{\text {het }}\left(\Omega-\Omega_{\mathrm{IF}}\right)-\bar{S}_{I I}^{\text {het }}\left(\Omega=\Omega_{\mathrm{IF}}^{-}\right)\right) \frac{d \Omega}{2 \pi}} \\
& =\frac{n_{\mathrm{tot}}+\frac{\beta-\alpha}{2}-C_{q q}+\frac{4 \Delta \Omega_{m}}{\kappa^{2}} C_{p p}}{n_{\mathrm{tot}}+\frac{\beta+\alpha}{2}+C_{q q}+\frac{4 \Delta \Omega_{m}}{\kappa^{2}} C_{p p}} \\
& =\frac{n_{\mathrm{tot}}+\left(\frac{4 \Delta \Omega_{m}}{\kappa^{2}} C_{p p}-C_{q q}\right)}{n_{\mathrm{tot}}+1+\left(\frac{4 \Delta \Omega_{m}}{\kappa^{2}} C_{p p}+C_{q q}\right)} .
\end{aligned}
$$

First, characteristic of linear detection, deviation of $R$ from unity in the ideal case $\left(C_{q q}=0=C_{p p}\right)$ is due to correlations developed between the quantum-backaction-driven mechanical motion and the detection process $[10,42]$. When $C_{q q}$ and $C_{p p}$ are finite, classical correlations are established that affect $R$. 
The response of the cavity (for $\Delta / \kappa \approx 0$ ) ensures that excess classical correlations due to input amplitude noise lead to an enhanced asymmetry, whereas those arising from input phase noise lead to a common increase in the sideband noise power.

\section{a. Expression for $\bar{S}_{y y}^{\text {het }}(\Omega)$}

In order to compare with Eq. (1), we identify the heterodyne spectrum Eq. (A23) with that of a positionequivalent heterodyne observable $y_{\text {het }}$, viz.,

$$
\begin{aligned}
& \bar{S}_{y y}^{\text {het }}\left(\Omega-\Omega_{\mathrm{IF}}\right)=\underbrace{\left(\frac{1}{4 C_{0} n_{c}}\right) \bar{S}_{x x}^{\mathrm{zp}}\left(\Omega_{m}\right)}_{\bar{S}_{x x}^{\text {imp.het }}(\Omega)} \\
& +\underbrace{\frac{\Gamma_{m}^{2}}{4}\left(\left|\chi_{m}[-\Omega]\right|^{2}+\left|\chi_{m}[\Omega]\right|^{2}\right)\left(n_{\text {tot }}+\frac{1}{2}\right) \bar{S}_{x x}^{\text {zp }}\left(\Omega_{m}\right)}_{\bar{S}_{x x}^{\text {tot }}(\Omega)} \\
& +\underbrace{\frac{\Gamma_{m}^{2}}{4}\left(\left|\chi_{m}[-\Omega]\right|^{2}\left(\frac{1}{2}+C_{q q}+\frac{4 \Delta \Omega_{m}}{\kappa^{2}} C_{p p}\right)+\left|\chi_{m}[\Omega]\right|^{2}\left(-\frac{1}{2}-C_{q q}+\frac{4 \Delta \Omega_{m}}{\kappa^{2}} C_{p p}\right)\right)}_{2 \operatorname{Re} \bar{S}_{v_{\text {tat }}^{\text {het }}} x_{\text {imp }}(\Omega)} .
\end{aligned}
$$

The identification is made by comparing the magnitude of the total thermal noise signal $\bar{S}_{x x}^{\text {tot }}$.

\section{b. Sensitivity of heterodyne and homodyne readout}

In the main text, frequent use is made of the phononequivalent sensitivity of the heterodyne and homodyne detectors. The sensitivity of balanced heterodyne detection (for the ideal case $\eta_{\text {het }}=1$ ), quantified as imprecision quanta,

$$
n_{\mathrm{imp}}^{\text {het }}=\left(4 \eta_{\mathrm{het}} C_{0} n_{c}\right)^{-1}
$$

is reduced by a factor of 4 compared to balanced homodyne detection (for the ideal case $\eta_{\text {hom }}=1$ ) of the phase quadrature of the output field, for which

$$
n_{\mathrm{imp}}^{\mathrm{hom}}=\left(16 \eta_{\mathrm{hom}} C_{0} n_{c}\right)^{-1} \text {. }
$$

This loss arises in equal part because (a) the heterodyne spectrum is double-sided and (b) both quadratures of the output field are detected.

\section{Measurement of excess laser noise}

\section{a. Excess amplitude noise}

In order to measure the noise in the amplitude quadrature, we employ direct photodetection of the probe laser (NewFocus Velocity). The measurement is made at the output of the tapered fiber, with the fiber retracted from the cavity. Analysis of the resulting photocurrent reveals the single-sided spectrum of the incident optical intensity (referred here for convenience to the incident optical power $\left.P=\hbar \omega_{L} \dot{n}\right)$

$$
\bar{S}_{P}(\Omega)=\left(\hbar \omega_{L}\right)^{2} \cdot 2 \bar{S}_{\dot{n} \dot{n}}(\Omega)=\left(\hbar \omega_{L}\right)^{2} \cdot 2\langle\dot{n}\rangle\left(1+2 C_{q q}\right) .
$$

A convenient characterization of the intensity noise is via the relative intensity noise (RIN) spectrum,

$$
\bar{S}_{\mathrm{RIN}}(\Omega):=\frac{\bar{S}_{P}(\Omega)}{\langle P\rangle^{2}},
$$

where excess amplitude noise manifests as a deviation from the shot-noise scaling $\propto 1 /\langle P\rangle$; more precisely,

$$
C_{q q}=\frac{1}{2}\left(\frac{\langle\dot{n}\rangle}{2} \bar{S}_{\mathrm{RIN}}(\Omega)-1\right) .
$$

Figure 5 shows an inference of $C_{q q}$ using Eq. (A31) and a measurement of $\bar{S}_{\text {RIN }}(\Omega)$ vs mean optical power. For

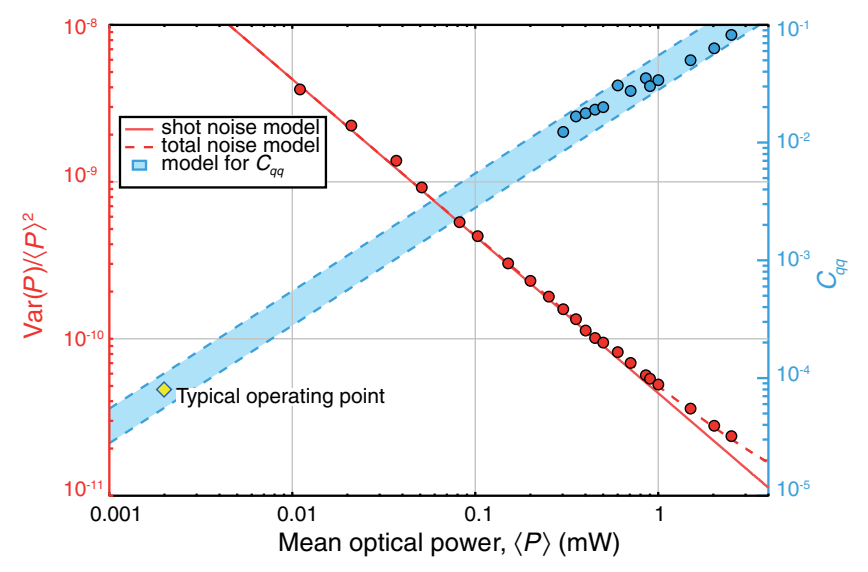

FIG. 5. Integrated (in a $100-\mathrm{kHz}$ band) relative intensity noise $\operatorname{Var}[P] /\langle P\rangle^{2}:=\int \bar{S}_{\mathrm{RIN}}\left(\Omega \approx \Omega_{m}\right)(d \Omega / 2 \pi)$ vs mean optical power. Deviation from shot-noise scaling is evident for $\langle P\rangle \gtrsim 1 \mathrm{~mW}$, attributed to classical amplitude noise. 


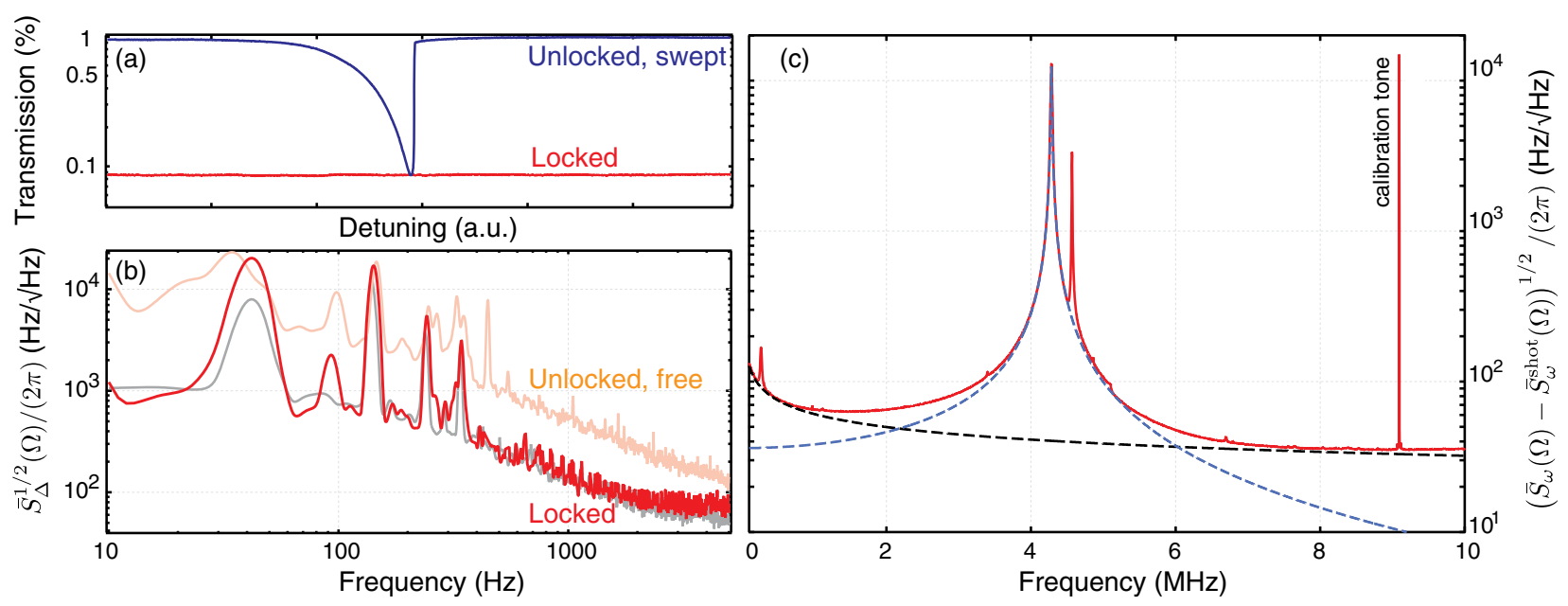

FIG. 6. (a) Residual detuning offset at dc estimated from the transmission signal when the laser is locked to the cavity. (b) Spectrum analysis of the lock error signal, generated via frequency-modulation spectroscopy [51], reveals low-frequency detuning jitter; when locked (red line), apparent detuning noise is limited by electronic noise (gray line) in the feedback loop, predominantly from the photodetector. (c) Excess frequency noise around the mechanical frequency inferred from a balanced homodyne measurement of the cavity output on resonance. The shot-noise-subtracted signal (red line) is composed of the thermomechanical motion of the mechanical mode (blue dashed line) and a contribution from excess frequency noise in the laser and cavity substrate (black dashed line).

typical experimental conditions $\quad(\langle P\rangle=1-5 \mu \mathrm{W})$, $C_{q q} \approx 10^{-4}$, so its contribution to sideband asymmetry is negligible.

Similar measurements performed on a Ti:Sa laser (MSquared Solstis) do not show deviation from shot-noise behavior even at $1 \mathrm{~mW}$; indeed, the ability to discern excess amplitude noise is limited by the saturation of our photodetectors beyond about $2 \mathrm{~mW}$ of incident power. The squeezing measurements in Fig. 2 are performed using the Ti:Sa laser.

\section{b. Excess phase noise}

Noise in the phase quadrature of the field leaking from the cavity is measured using balanced homodyne detection. This signal reveals phase noise originating from the input laser as well as apparent phase noise from the cavity. Referred to as cavity frequency noise, the homodyne photocurrent spectral density is given by

$$
\begin{aligned}
\bar{S}_{\omega}(\Omega)= & \Omega^{2} \bar{S}_{\phi}(\Omega) \\
= & \Omega^{2}\left[\bar{S}_{\phi}^{\mathrm{in}, \mathrm{shot}}(\Omega)+\bar{S}_{\phi}^{\mathrm{in}, \mathrm{ex}}(\Omega)\right. \\
& \left.+\bar{S}_{\phi}^{\mathrm{cav}, \mathrm{ex}}(\Omega)+\bar{S}_{\phi}^{\mathrm{cav}, \operatorname{mech}}(\Omega)\right] .
\end{aligned}
$$

Here, $\bar{S}_{\omega}$ contains contributions from laser phase noise (shot and excess), cavity substrate noise (including thermorefractive [48,49] and thermomechanical noise [50]) and thermal motion of other modes of the mechanical resonator. The total excess noise in the phase quadrature is modeled by $C_{p p}$, which allows us to infer the latter using

$$
\frac{C_{p p}}{\langle\dot{n}\rangle}=\bar{S}_{\phi}^{\text {in,ex }}\left(\Omega_{m}\right)+\bar{S}_{\phi}^{\text {cav,ex }}\left(\Omega_{m}\right)
$$

Figure 6(c) shows a homodyne measurement made with $3 \mathrm{~mW}$ of local oscillator power, whose shot noise has been subtracted. The spectrum is calibrated by referencing it against a known phase modulation tone injected at the input of the homodyne interferometer [52]. The total excess frequency noise (red line) is dominated by thermal motion of the in-plane and out-of-plane modes, both of which are gas damped for this measurement. A joint fit to (a) a model of a velocity-damped oscillator (blue dashed line) and (b) a model combining thermorefractive $[48,49]$ and white frequency noise (black dashed line), gives an estimate of $\bar{S}_{\omega}^{\mathrm{ex}}(\Omega)$. Frequency noise intrinsic to the diode laser was independently measured using an imbalanced interferometer, consistent with the model used to fit the total observed frequency noise. Near the mechanical frequency, $\bar{S}_{\omega}^{\mathrm{ex}}\left(\Omega_{m}\right) \approx 2 \pi(35 \mathrm{~Hz} / \sqrt{\mathrm{Hz}})^{2}$, implying [via Eq. (A33)] $C_{p p} \approx 30$ (using signal power of $\approx 100 \mathrm{nW}$ ).

From this estimate of $C_{p p}$, we are able to bound two quantities. First, in conjunction with $C_{q q} \ll 0.01$, the excess noise cross-correlation is bounded as $C_{q p} \ll 1$. Second, referring to Eq. (A23), we are able to estimate the contribution of phase noise to the heterodyne sideband. This contribution, characterized as an equivalent phonon occupation (since it adds positive noise power to either sideband),

$$
n_{\phi}=\frac{\Delta}{\kappa} \frac{4 \Omega_{m}}{\kappa} C_{p p},
$$

has a mean value determined by the mean offset in the detuning $\bar{\Delta}$. Figure 6 (a) allows an estimate, $\bar{\Delta} \approx 0.01 \cdot \kappa$, giving 


$$
\begin{aligned}
\bar{n}_{\phi} & =\frac{\bar{\Delta}}{\kappa} \frac{4 \Omega_{m}}{\kappa} C_{p p} \\
& =0.0052 \cdot\left(\frac{\bar{\Delta} / \kappa}{0.01}\right) 4\left(\frac{\Omega_{m} / 2 \pi}{4.3 \mathrm{MHz}}\right)\left(\frac{1 \mathrm{GHz}}{\kappa / 2 \pi}\right)\left(\frac{C_{p p}}{30}\right) .
\end{aligned}
$$

Low-frequency detuning noise $\delta \Delta$ [Fig. 6(b)] causes deviations from this mean, which are significant if their effect is comparable to $\bar{n}_{\phi}$. We bound the probability for such "large" statistical excursions using Chebyshev's inequality [53],

$$
\begin{aligned}
\operatorname{Pr}\left(\left|n_{\phi}-\bar{n}_{\phi}\right|>\bar{n}_{\phi}\right) & \leq \frac{\operatorname{Var}\left[n_{\phi}\right]}{\bar{n}_{\phi}^{2}} \\
& =\left(\frac{4 \Omega_{m}}{\kappa} \frac{C_{p p}}{\bar{n}_{\phi}}\right)^{2} \frac{\operatorname{Var}[\delta \Delta]}{\kappa^{2}} \\
& \approx 10^{-6} .
\end{aligned}
$$

We thus estimate that mean residual detuning is the leading contribution to phase noise contamination; however, the contamination, characterized as a phononequivalent noise power $\bar{n}_{\phi}=0.005$, is an insignificant contribution to the sideband ratio Eq. (A25).

\section{Bounding the value of the classical noise cross-correlation $C_{q p}$}

In Ref. [37], excess classical noise in the laser is modeled as an independent classical stochastic process introduced explicitly into $\delta a_{\text {in }}$. The added term, being a classical stochastic process, obeys a Cauchy-Schwarz inequality for its second moments, resulting in the inequality $C_{q p} \leq \sqrt{C_{q q} C_{p p}}$, which may be employed to bound the magnitude of $C_{q p}$, given measurements of $C_{q q}$ and $C_{p p}$.

Here, we consider a more natural alternative, where the ansatz Eq. (A6) is supposed to arise from a choice of the underlying quantum state that models the classical component of the noise. From this perspective, the ansatz in Eq. (A6) is a valid one as long as it arises from a legitimate quantum state $\rho$. The sufficient condition for the matrix in Eq. (A6) to be a valid covariance matrix is [54]

$$
V:=\left(\begin{array}{cc}
\frac{1}{2}+C_{q q} & C_{q p} \\
C_{q p} & \frac{1}{2}+C_{p p}
\end{array}\right) \geq 0 .
$$

In particular, this implies that $\operatorname{Tr} V \geq 0$ and $\operatorname{det} V \geq 0$; the latter condition gives

$$
\begin{aligned}
C_{q p}^{2} & \leq C_{q q} C_{p p}+\frac{1}{2}\left(C_{q q}+C_{p p}\right) \\
& \leq C_{q q} C_{p p}+\sqrt{C_{q q} C_{p p}} \\
& =C_{q q} C_{p p}\left(1+\frac{1}{\sqrt{C_{q q} C_{p p}}}\right) .
\end{aligned}
$$

Here, the second line is obtained by employing the inequality $C_{q q}+C_{p p} \geq 2 \sqrt{C_{q q} C_{p p}}$, which generally follows from the fact that $C_{q q, p p}$ are positive.

Ultimately, in the limit $C_{q q} C_{p p} \gg 1$, we recover the result in Ref. [37], namely, $C_{q p} \leq\left(C_{q q} C_{p p}\right)^{1 / 2}$; however, in the opposite limit, $C_{q q} C_{p p} \ll 1$, the appropriate bound is $C_{q p} \leq\left(C_{q q} C_{p p}\right)^{1 / 4}$, so employing the conventional Cauchy-Schwarz inequality would lead to an underestimate of $C_{q p}$.

In our case, $C_{q q} C_{p p} \approx 3 \times 10^{-3}$, and Eq. (A38) suggests $C_{q p} \ll 1$.

\section{APPENDIX B: SQUEEZING IN HOMODYNE DETECTION}

In the experimentally relevant bad cavity regime, $\Omega_{m} \ll \kappa$, resonant probing $\Delta=0$, and quantum-noise limited probe laser, a significantly simplified analysis illustrates the presence of correlations in the transmitted field.

Following from Eqs. (A16) and (A17), the cavity transmission is given by

$\delta a_{\mathrm{out}}[\Omega] \approx-\delta a_{\mathrm{in}}[\Omega]-i \frac{\sqrt{C_{0} n_{c} \Gamma_{m}}}{x_{\mathrm{zp}}}\left(x_{\mathrm{th}}[\Omega]+x_{\mathrm{ba}}[\Omega]\right)$,

where the total motion, $x:=x_{\mathrm{zp}}\left(b+b^{\dagger}\right)$, has been partitioned into the (intrinsic) thermal motion $x_{\text {th }}$ because of the ambient environment,

$\delta x_{\mathrm{th}}[\Omega]:=x_{\mathrm{zp}} \sqrt{\Gamma_{m}}\left(\chi_{m}[\Omega] \delta b_{\text {in }}[\Omega]+\chi_{m}[-\Omega]^{*} \delta b_{\text {in }}^{\dagger}[\Omega]\right)$,

and $x_{\mathrm{ba}}$, the backaction-driven motion,

$$
\begin{aligned}
\delta x_{\mathrm{ba}}[\Omega]: & :=x_{\mathrm{zp}} \sqrt{2 C_{0} n_{c} \Gamma_{m}} \frac{2 \Omega_{m}}{\mathcal{N}[\Omega]} \delta q_{\mathrm{in}}[\Omega] \\
& \approx x_{\mathrm{zp}} \sqrt{2 C_{0} n_{c} \Gamma_{m}} \frac{\delta q_{\mathrm{in}}[\Omega]}{\left(\Omega-\Omega_{m}\right)-i\left(\Gamma_{m} / 2\right)}
\end{aligned}
$$

because of the vacuum fluctuations in the amplitude quadrature of the input optical field. Note that the second equality neglects dynamical backaction and assumes a high- $Q$ mechanical oscillator.

In terms of the amplitude $(\delta q)$ and phase $(\delta p)$ quadratures, Eq. (B1) takes the form 


$$
\begin{aligned}
\delta q_{\text {out }}[\Omega]= & -\delta q_{\text {in }}[\Omega], \\
\delta p_{\text {out }}[\Omega]= & -\delta p_{\text {in }}[\Omega]-\frac{\sqrt{2 C_{0} n_{c} \Gamma_{m}}}{x_{\mathrm{zp}}}\left(x_{\text {th }}[\Omega]+x_{\text {ba }}[\Omega]\right) \\
= & -\delta p_{\text {in }}[\Omega]-\sqrt{2 C_{0} n_{c} \Gamma_{m}} \frac{x_{\text {th }}[\Omega]}{x_{\mathrm{zp}}} \\
& -\frac{2 C_{0} n_{c} \Gamma_{m}}{\left(\Omega-\Omega_{m}\right)-i\left(\Gamma_{m} / 2\right)} \delta q_{\text {in }}[\Omega] .
\end{aligned}
$$

Note that the transmitted phase quadrature has a component proportional to the transmitted amplitude quadrature, leading to phase-amplitude correlations described by the (unsymmetrized, double-sided) cross-correlation spectrum,

$$
S_{p q}^{\text {out }}(\Omega)=-\frac{i}{2}+\frac{C_{0} n_{c} \Gamma_{m}}{\left(\Omega-\Omega_{m}\right)-i\left(\Gamma_{m} / 2\right)},
$$

where the first term is due to the commutation relation of the transmitted fields, while the second arises from correlations induced by the optomechanical interaction.

Homodyne detection of the phase quadrature, corresponding to a measurement of $\delta p_{\text {out }}$ alone, does not give access to these optomechanically induced correlations. However, homodyne detection at a finite phase offset $\theta$, corresponding to a measurement of

$$
\delta q_{\text {out }}^{\theta}[\Omega]:=\delta q_{\text {out }}[\Omega] \cos \theta+\delta p_{\text {out }}[\Omega] \sin \theta,
$$

can directly access amplitude-phase correlations. Indeed, the homodyne photocurrent spectrum, $\bar{S}_{I I}^{\text {hom }, \theta}(\Omega) \propto \bar{S}_{q q}^{\text {out }, \theta}(\Omega)$, takes the form

$$
\begin{gathered}
\bar{S}_{I I}^{\text {hom }, \theta}(\Omega) \propto \\
+\cos { }^{2} \theta \bar{S}_{q q}^{\text {out }}(\Omega)+\sin ^{2} \theta \bar{S}_{p p}^{\text {out }}(\Omega) \\
+\sin (2 \theta) \operatorname{Re} S_{p q}^{\text {out }}(\Omega),
\end{gathered}
$$

so for $\theta \neq 0, \pi / 2$, the correlation term is manifest. Including the effect of nonideal detection efficiency, $\eta_{\text {hom }} \leq 1$, and normalizing to shot noise, the homodyne photocurrent spectrum is

$$
\begin{aligned}
\bar{S}_{I I}^{\text {hom }, \theta}(\Omega)= & 1+4 C_{0} n_{c} \eta_{\text {hom }} \frac{\bar{S}_{x x}(\Omega)}{x_{\mathrm{zp}}^{2}} \sin ^{2} \theta \\
& +\underbrace{2 C_{0} n_{c} \eta_{\mathrm{hom}} \frac{\Gamma_{m}\left(\Omega-\Omega_{m}\right)}{\left(\Omega-\Gamma_{m}\right)^{2}\left(\Gamma_{m} / 2\right)^{2}} \sin (2 \theta)}_{2 \operatorname{Re} \bar{S}_{x_{\mathrm{ba}} x_{\mathrm{imp}}}^{\text {hom }}(\Omega)} .
\end{aligned}
$$

The last term, antisymmetric in frequency about the mechanical resonance frequency $\Omega_{m}$, can contribute negatively to the photocurrent spectrum, leading to squeezing below the shot-noise level. The last term can be identified as being due to correlations between the backaction-driven motion $x_{\mathrm{ba}}$ and the fluctuations of the transmitted field that set the imprecision in homodyne detection. The above equation is used to fit the squeezing spectrum in Fig. 2.

\section{Relation to heterodyne sideband asymmetry}

Following the discussion of heterodyne detection in Sec. A 1, leading up to Eqs. (A21) and (A22), the heterodyne photocurrent spectrum centered around the intermediate frequency $\Omega_{\mathrm{IF}}$ is given by

$$
\begin{aligned}
\bar{S}_{I I}^{\text {het }}\left(\Omega-\Omega_{\mathrm{IF}}\right) \propto & \bar{S}_{q q}^{\text {out }}(\Omega)+\bar{S}_{p p}^{\text {out }}(\Omega) \\
& +\operatorname{Im}\left(S_{q p}^{\text {out }}(-\Omega)-S_{p q}^{\text {out }}(+\Omega)\right),
\end{aligned}
$$

where $\Omega \geq 0$ and the approximation $\Omega_{\mathrm{IF}} \gg \Omega_{m} \gg 0$ is used. Indeed, the asymmetry in the heterodyne spectrum, about $\Omega=\Omega_{\mathrm{IF}}$, arises from the imaginary part of the quantum correlations between the phase and amplitude of the transmitted field. Compared to the analogous expression for the homodyne photocurrent spectrum in Eq. (B7), where the real part of the correlation leads to optical squeezing, it is the imaginary part of the phaseamplitude correlation [Eq. (B5)] that contributes to sideband asymmetry.

\section{APPENDIX C: DISPLACEMENT SPECTRUM OF A COLD-DAMPED MECHANICAL OSCILLATOR}

Here, we recall a few useful expressions for the displacement spectrum of a cold-damped mechanical oscillator $[18,38]$. We denote by $x$ the physical displacement of the oscillator and by $y_{\text {hom }}=x+x_{\text {imp }}^{\text {hom }}$ the apparent displacement measured at the in-loop (homodyne) detector. Following the arguments detailed in the supplementary information of Ref. [18], we get

$$
\begin{aligned}
\bar{S}_{x}(\Omega)= & \underbrace{\left|\chi_{\text {eff }}(\Omega)\right|^{2}\left(2 n_{\text {tot }}+1\right) \bar{S}_{x}^{\mathrm{zp}}\left(\Omega_{\mathrm{m}}\right)}_{\bar{S}_{x}^{\text {tot }}(\Omega)} \\
& +\underbrace{\left|\chi_{\mathrm{eff}}(\Omega)\right|^{2}\left(2 n_{\mathrm{imp}}^{\mathrm{hom}} g_{\mathrm{fb}}^{2}\right) \bar{S}_{x}^{\mathrm{zp}}\left(\Omega_{\mathrm{m}}\right)}_{\bar{S}_{x}^{\mathrm{tb}}(\Omega)}
\end{aligned}
$$

for the physical displacement spectrum and

$$
\begin{aligned}
\bar{S}_{y}^{\text {hom }}(\Omega)= & \underbrace{2 n_{\text {imp }}^{\text {hom }} \bar{S}_{x}^{\text {zp }}\left(\Omega_{\mathrm{m}}\right)}_{\bar{S}_{x}^{\text {imp.hom }}(\Omega)} \\
& +\underbrace{\left|\chi_{\text {eff }}(\Omega)\right|^{2}\left(2 n_{\text {tot }}+1\right) \bar{S}_{x}^{\text {zp }}\left(\Omega_{\mathrm{m}}\right)}_{\bar{S}_{x}^{\text {tot }}(\Omega)} \\
& +\underbrace{\left|\chi_{\text {eff }}(\Omega)\right|^{2}\left(-2 n_{\text {imp }}^{\text {hom }} g_{\mathrm{fb}}\right) \bar{S}_{x}^{\text {zp }}\left(\Omega_{\mathrm{m}}\right)}_{2 \operatorname{Re}_{x_{\mathrm{fb}} x_{\text {imp }}^{\text {hom }}}(\Omega)}
\end{aligned}
$$

for the apparent displacement spectrum. Here, the effective susceptibility for the displacement is given by 


$$
\chi_{\mathrm{eff}}=\frac{\Omega_{m} \Gamma_{m}}{\left(\Omega_{m}^{2}-\Omega^{2}\right)+i \Omega\left(\Gamma_{m}+\Gamma_{\mathrm{fb}}\right)},
$$

where $\Gamma_{\mathrm{fb}}=\Gamma_{m} g_{\mathrm{fb}}$ is the feedback damping rate. In the main text, we use the approximation $\Gamma_{m}+\Gamma_{\mathrm{fb}} \approx \Gamma_{\mathrm{fb}}$.

[1] H. M. Wiseman and G. J. Milburn, Quantum Measurement and Control (Cambridge University Press, Cambridge, 2010).

[2] V. Braginsky and F. Khalili, Quantum Measurement (Cambridge University Press, Cambridge, 1992).

[3] A. A. Clerk, M. H. Devoret, S. M. Girvin, F. Marquardt, and R.J. Schoelkopf, Introduction to Quantum Noise, Measurement, and Amplification, Rev. Mod. Phys. 82, 1155 (2010).

[4] LIGO Scientific Collaboration and Virgo Collaboration, GW150914: The Advanced LIGO Detectors in the Era of First Discoveries, Phys. Rev. Lett. 116, 131103 (2016).

[5] D. W. C. Brooks, T. Botter, S. Schreppler, T. P. Purdy, N. Brahms, and D. M. Stamper-Kurn, Non-Classical Light Generated by Quantum-Noise-Driven Cavity Optomechanics, Nature (London) 488, 476 (2012).

[6] A. H. Safavi-Naeini, S. Gröblacher, J. T. Hill, J. Chan, M. Aspelmeyer, and O. Painter, Squeezed Light from a Silicon Micromechanical Resonator, Nature (London) 500, 185 (2013).

[7] T. P. Purdy, P. Yu, R. W. Peterson, N. S. Kampel, and C. A. Regal, Strong Optomechanical Squeezing of Light, Phys. Rev. X 3, 031012 (2013).

[8] W. H. P. Nielsen, Y. Tsaturyan, C. B. Moller, E. S. Polzik, and A. Schliesser, Multimode Optomechanical System in the Quantum Regime, arXiv:1605.06541.

[9] A. H. Safavi-Naeini, J. Chan, J. T. Hill, T. P. M. Alegre, A. Krause, and O. Painter, Observation of Quantum Motion of a Nanomechanical Resonator, Phys. Rev. Lett. 108, 033602 (2012).

[10] A. J. Weinstein, C. U. Lei, E. E. Wollman, J. Suh, A. Metelmann, A. A. Clerk, and K. C. Schwab, Observation and Interpretation of Motional Sideband Asymmetry in a Quantum Electromechanical Device, Phys. Rev. X 4, 041003 (2014).

[11] T. P. Purdy, P.-L. Yu, N. S. Kampel, R. W. Peterson, K. Cicak, R. W. Simmonds, and C. A. Regal, Optomechanical Raman-Ratio Thermometry, Phys. Rev. A 92, 031802 (2015).

[12] M. Underwood, D. Mason, D. Lee, H. Xu, L. Jiang, A. B. Shkarin, K. Borkje, S. M. Girvin, and J. G. E. Harris, Measurement of the Motional Sidebands of a NanogramScale Oscillator in the Quantum Regime, Phys. Rev. A 92, 061801 (2015).

[13] J. H. Shapiro, Quantum Noise and Excess Noise in Optical Homodyne and Heterodyne Receivers, IEEE J. Quantum Electron. 21, 237 (1985).

[14] F. Khalili, H. Miao, H. Yang, A. H. Safavi-Naeini, O. Painter, and Y. Chen, Quantum Back-Action in Measurements of Zero-Point Mechanical Oscillations, Phys. Rev. A 86, 033840 (2012).
[15] L. F. Buchmann, S. Schreppler, J. Kohler, N. Spethmann, and D. M. Stamper-Kurn, Complex Squeezing and Force Measurement Beyond the Standard Quantum Limit, Phys. Rev. Lett. 117, 030801 (2016).

[16] Note that sideband asymmetry arising for direct photon counting of the meter field has a different origin [10,17].

[17] M. O. Scully and S. Stenholm, The Role of Vacuum Fluctuations and Spontaneous Emission in the Laser Linewidth, Phys. Scr. T21, 119 (1988).

[18] D. J. Wilson, V. Sudhir, N. Piro, R. Schilling, A. Ghadimi, and T. J. Kippenberg, Measurement-Based Control of a Mechanical Oscillator at Its Thermal Decoherence Rate, Nature (London) 524, 325 (2015).

[19] J. M. W. Milatz, J. J. Van Zolingen, and B. B. Van Iperen, The Reduction in the Brownian Motion of Electrometers, Physica (Amsterdam) 19, 195 (1953).

[20] R. L. Forward, Electronic Cooling of Resonant Gravity Gradiometers, J. Appl. Phys. 50, 1 (1979).

[21] P. Cohadon, A. Heidmann, and M. Pinard, Cooling of a Mirror by Radiation Pressure, Phys. Rev. Lett. 83, 3174 (1999).

[22] B. D’Urso, B. Odom, and G. Gabrielse, Feedback Cooling of a One-Electron Oscillator, Phys. Rev. Lett. 90, 043001 (2003).

[23] D. Kleckner and D. Bouwmeester, Sub-Kelvin Optical Cooling of a Micromechanical Resonator, Nature (London) 444, 75 (2006).

[24] M. Poggio, C. Degen, H. Mamin, and D. Rugar, Feedback Cooling of a Cantilever's Fundamental Mode below $5 \mathrm{mK}$, Phys. Rev. Lett. 99, 017201 (2007).

[25] T. Corbitt, C. Wipf, T. Bodiya, D. Ottaway, D. Sigg, N. Smith, S. Whitcomb, and N. Mavalvala, Optical Dilution and Feedback Cooling of a Gram-Scale Oscillator to 6.9 mK, Phys. Rev. Lett. 99, 160801 (2007).

[26] A. Vinante et al., Feedback Cooling of the Normal Modes of a Massive Electromechanical System to Submillikelvin Temperature, Phys. Rev. Lett. 101, 033601 (2008).

[27] B. Abbott et al., Observation of a Kilogram-Scale Oscillator Near Its Quantum Ground State, New J. Phys. 11, 073032 (2009).

[28] A. Kubanek, M. Koch, C. Sames, A. Ourjoumtsev, P. W. H. Pinkse, K. Murr, and G. Rempe, Photon-by-Photon Feedback Control of a Single-Atom Trajectory, Nature (London) 462, 898 (2009).

[29] T. Li, S. Kheifets, and M. G. Raizen, Millikelvin Cooling of an Optically Trapped Microsphere in Vacuum, Nat. Phys. 7, 527 (2011).

[30] J. Giesler, B. Deutsch, R. Quidant, and L. Novotny, Subkelvin Parametric Feedback Cooling of a Laser-Trapped Nanoparticle, Phys. Rev. Lett. 109, 103603 (2012).

[31] M. Hatridge, S. Shankar, M. Mirrahimi, F. Shackert, K. Geerlings, T. Brecht, K. Sliwa, B. Abdo, L. Frunzio, S. M. Girvin, R. J. Schoelkopf, and M. H. Devoret, Quantum Back-Action of an Individual Variable-Strength Measurement, Science 339, 178 (2013).

[32] M. S. Taubman, H. M. Wiseman, D. E. McClelland, and H. A. Bachor, Intensity Feedback Effects on QuantumLimited Noise, J. Opt. Soc. Am. B 12, 1792 (1995).

[33] R. W. Peterson, T. P. Purdy, N. S. Kampel, R. W. Andrews, P.-L. Yu, K. W. Lehnert, and C. A. Regal, Laser Cooling of a 
Micromechanical Membrane to the Quantum Backaction Limit, Phys. Rev. Lett. 116, 063601 (2016).

[34] Here, heterodyne spectra are expressed as double-sided symmetrized spectra, for example, $\bar{S}_{y y}$, while homodyne spectra are expressed as the corresponding single-sided symmetrized versions, for example, $\bar{S}_{y}$.

[35] R. Schilling, H. Schütz, A. H. Ghadimi, V. Sudhir, D. J. Wilson, and T. J. Kippenberg, Near-Field Integration of a SiN Nanobeam and a $\mathrm{SiO}_{2}$ Microcavity for HeisenbergLimited Displacement Sensing, Phys. Rev. Applied 5, 054019 (2016).

[36] C. Fabre, M. Pinard, S. Bourzeix, A. Heidmann, E. Giacobino, and S. Reynaud, Quantum-Noise Reduction Using a Cavity with a Movable Mirror, Phys. Rev. A 49, 1337 (1994).

[37] A. M. Jayich, J. C. Sankey, K. Borkje, D. Lee, C. Yang, M. Underwood, L. Childress, A. Petrenko, S. M. Girvin, and J. G. E. Harris, Cryogenic Optomechanics with a $\mathrm{Si}_{3} \mathrm{~N}_{4}$ Membrane and Classical Laser Noise, New J. Phys. 14, 115018 (2012).

[38] J. M. Courty, A. Heidmann, and M. Pinard, Quantum Limits of Cold Damping with Optomechanical Coupling, Eur. Phys. J. D 17, 399 (2001).

[39] S. Mancini, D. Vitali, and P. Tombesi, Optomechanical Cooling of a Macroscopic Oscillator by Homodyne Feedback, Phys. Rev. Lett. 80, 688 (1998).

[40] H. M. Wiseman, Squashed States of Light: Theory and Applications to Quantum Spectroscopy, J. Opt. B 1, 459 (1999).

[41] A. A. Clerk, F. Marquardt, and K. Jacobs, Back-Action Evasion and Squeezing of a Mechanical Resonator Using a Cavity Detector, New J. Phys. 10, 095010 (2008).

[42] A. H. Safavi-Naeini, J. Chan, J. T. Hill, S. Gröblacher, H. Miao, Y. Chen, M. Aspelmeyer, and O. Painter, Laser Noise in Cavity-Optomechanical Cooling and Thermometry, New J. Phys. 15, 035007 (2013).

[43] In addition, it is known that for semiconductor lasers, phaseamplitude correlations are limited to frequencies close to their relaxation oscillation frequency $[44,45]$; the latter is typically at a few $\mathrm{GHz}$ from the carrier [46] - irrelevant for our experiment.

[44] K. Vahala, C. Harder, and A. Yariv, Observation of Relaxation Resonance Effects in the Field Spectrum of Semiconductor Lasers, Appl. Phys. Lett. 42, 211 (1983).

[45] M. van Exeter, W. Hamel, J. P. Woerdman, and B. Zeijlmans, Spectral Signature of Relaxation Oscillations in Semiconductor Lasers, IEEE J. Quantum Electron. 28, 1470 (1992).

[46] T. J. Kippenberg, A. Schliesser, and M. L. Gorodetsky, Phase Noise Measurement of External Cavity Diode Lasers and Implications for Optomechanical Sideband Cooling of $\mathrm{GHz}$ Mechanical Modes, New J. Phys. 15, 015019 (2013).

[47] M. Aspelmeyer, T. J. Kippenberg, and F. Marquardt, Cavity Optomechanics, Rev. Mod. Phys. 86, 1391 (2014).

[48] V. B. Braginsky, M. L. Gorodetsky, and S. P. Vyatchanin, Thermo-Refractive Noise in Gravitational Wave Antennae, Phys. Lett. A 271, 303 (2000).

[49] M. L. Gorodetsky and I. S. Grudinin, Fundamental Thermal Fluctuations in Microspheres, J. Opt. Soc. Am. B 21, 697 (2004).

[50] A. Gillespie and F. Raab, Thermal Noise in the Test Mass Suspensions of a Laser Interferometer Gravitational-Wave Detector Prototype, Phys. Lett. A 178, 357 (1993).

[51] G.C. Bjorklund, Frequency-Modulation Spectroscopy: A New Method for Measuring Weak Absorptions and Dispersions, Opt. Lett. 5, 15 (1980).

[52] M. L. Gorodetsky, A. Schliesser, G. Anetsberger, S. Deleglise, and T.J. Kippenberg, Determination of the Vacuum Optomechanical Coupling Rate Using Frequency Noise Calibration, Opt. Express 18, 23236 (2010).

[53] H. Cramer, Mathematical Methods of Statistics (Princeton University Press, Princeton, NJ, 1946).

[54] R. Simon, N. Mukunda, and B. Dutta, Quantum-Noise Matrix for Multimode Systems: U(n) Invariance, Squeezing, and Normal Forms, Phys. Rev. A 49, 1567 (1994). 